\title{
Optimal Treatment for Patients with Oligometastatic Prostate Cancer
}

\author{
Bulent Cetin $^{a}$ Chiara A. Wabl $^{\mathrm{b}}$ Ozge Gumusay ${ }^{\mathrm{b}}$ \\ ${ }^{a}$ Division of Medical Oncology, Department of Internal Medicine, Suleyman Demirel University Faculty of Medicine,

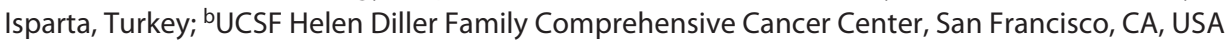

\section{Keywords}

Oligometastatic disease · Prostate cancer · Local treatment · Systemic therapy

\begin{abstract}
Oligometastatic prostate cancer (PCa) can be defined as cancer with a limited number of metastases, typically fewer than 5 lesions, and involves lesions contained within the axial versus the appendicular skeleton. Patients can present with de novo oligometastatic, oligorecurrent, or oligoprogressive PCa. Oligometastatic PCa patients demonstrate considerable improvements in survival outcomes, with a better prognosis than patients with extensive metastatic disease. However, the management of patients that present with nonsymptomatic oligometastatic PCa remains difficult. In the oligometastatic setting, the benefit of local therapies such as prostatectomy and radiotherapy on survival outcomes is an intriguing topic; however, their impact on oncological outcomes is still unknown.

(C) 2021 S. Karger AG, Basel
\end{abstract}

\section{Introduction}

Prostate cancer $(\mathrm{PCa})$ is the most commonly diagnosed life-threatening cancer in men (164,690 cases and 26,120 deaths in 2018) [1]. One in 5 patients develops metastatic (M1) disease. Incidence rates of M1 PCa have increased ap-

preciably although slowly in the past 10 years, with rates among men aged $45-54$ years increasing since 2004 [2]. The absolute number of new cases of M1 PCa (annual burden) is projected to increase by $42 \%$ over the next decade, with 15,097 new cases expected in 2025. Many questions regarding disease management remain: what is the best way to document and characterize disease burden? Once we have identified and characterized a lesion, what are the best ways to approach these sites of disease? If a primary tumor remains untreated, should it be treated? What should the systemic treatment be in cases of oligometastatic disease? In this review, we will discuss oligometastatic PCa scenarios, and we intend to provide the reader with an evidence-based guide of treatment decisions (Table 1).

\section{Defining Oligometastatic Disease}

\section{Clinical Scenario 1}

A 55-year-old male with a prostate-specific antigen (PSA) of $33 \mathrm{ng} / \mathrm{dL}$ has Gleason $4+5$ adenocarcinoma of the prostate diagnosed on a biopsy. He is generally well and has no relevant medical history. Computed tomography (CT) and bone scans show evidence of metastasis throughout the pelvis. In your opinion, which terminology best describes M1 PCa in patients who are about to start treatment?

The concept of oligometastatic disease was first proposed by Sam Hellman [3] and Ralph Weichselbaum [4] 
Table 1. Oligometastatic PCa

\begin{tabular}{|c|c|c|}
\hline । & Multidisciplinary discussion and careful planning & How many lesions and where? \\
\hline II & Synchronous disease & $\begin{array}{l}\text { Does local treatment of all visualized disease impact patients or disease-related outcomes? } \\
\text { Has the primary tumor been treated? }\end{array}$ \\
\hline III & Metachronous or progressive disease & Does MDT impact patients or disease-related outcomes? \\
\hline V & Which disease sites & Tumor burden spectrum: minimal to widespread disease on conventional imaging \\
\hline $\mathrm{VI}$ & Which patients & $\begin{array}{l}\text { Do all metastatic patients equally benefit from the primary treatment in oligometastatic } \\
\text { diseases? }\end{array}$ \\
\hline IX & How to incorporate new therapies & \\
\hline \multirow[t]{3}{*}{$\mathrm{x}$} & What are validated endpoints & Impact OS versus delay ADT (STOMP and ORIOLE) \\
\hline & & Time to polymetastatic progression \\
\hline & & Time to systemic therapy \\
\hline
\end{tabular}

MDT, metastasis-directed therapy; PCa, prostate cancer; ADT, androgen deprivation therapy.

at the American Society of Clinical Oncology (ASCO) over 20 years ago. It is derived from the Greek word "oligo" meaning "few." The concept involves controlling a primary tumor completely or controlling a single or limited number of M1 lesions with local therapy, which can result in a cure. Oligometastatic disease is an intermediate biological state with a unique clinical picture within the spectrum of advanced disease, which has a favorable phenotype that is ideal for an intensive approach. This disease state will continue to be redefined as novel imaging tools are adopted as it remains relatively poorly understood. The oligometastatic concept was developed based on data from gastrointestinal-associated liver metastasis: resection of M1 liver lesions in patients with gastrointestinal malignancy is curative. When considering whether a patient has oligometastatic disease, it is important to think about the following: first, if the primary tumor and M1 lesions are controlled and the patient still progresses, the patient did not actually have oligometastatic cancer. Second, oligometastatic cancer treatment should not require any systemic therapy. Lastly, the definition and diagnosis of oligometastatic disease is independent of timing (synchronous vs. metachronous).

The definition of oligometastatic PCa has evolved over time. It was first noted by Soloway et al. [5] that individuals who had a limited number of lesions on bone scans had improved survival outcomes compared to those who presented with bulky disease. This was confirmed in a European study by Ost et al. [6], where individuals who presented with a single M1 site had significantly improved 5-year survival outcomes compared to those who had polymetastatic disease. Gandaglia et al. [7] demon- strated that the survival rates varied depending on the site of disease and the number of metastases in the 3,857 patients presenting with M1 PCa. Visceral metastases alone or with concomitant bone involvement predicted the worst survival outcome compared with bone metastases alone. A retrospective cohort of 436 patients with M1 castration-sensitive PCa (mCSPC) categorized patients into 2 groups at the start of androgen deprivation therapy (ADT): those with de novo disease or prior local therapy and those with high volume or low volume of disease [8]. De novo/high-volume patients showed a robust, $>2$-fold higher risk of developing castration-resistant $\mathrm{PCa}$ (hazard ratio $[\mathrm{HR}]=2.09 ; 95 \% \mathrm{CI}: 1.63-2.66)$ or death $(\mathrm{HR}=$ $2.48 ; 95 \%$ CI: 1.83-3.36). The results from Sridharan et al. [9] indicated that at the time of the first M1 diagnosis, patients with oligometastatic bone disease at either 1 site only or at 2-3 sites experienced longer time intervals to $\mathrm{PCa}$-specific death than patients who were diagnosed with 4 or more bone metastases. Our modern take on oligometastatic PCa came out of an analysis from the SWOG 8494 study, from which researchers began to provide some granularity to this definition [10]. In SWOG 8494, patients with low-volume disease were defined as those with axial metastasis and/or lymph node metastasis only, versus high-volume disease, which included patients with appendicular metastases and/or visceral metastases. Presently, there are a tremendous number of ongoing and retrospective trials that use various definitions for oligometastatic disease. There is no true consensus on the definition, but most ongoing studies define oligometastatic PCa as having a limited number of M1 sites (Fig. 1). Typically, these studies include fewer than 5 lesions; ex- 


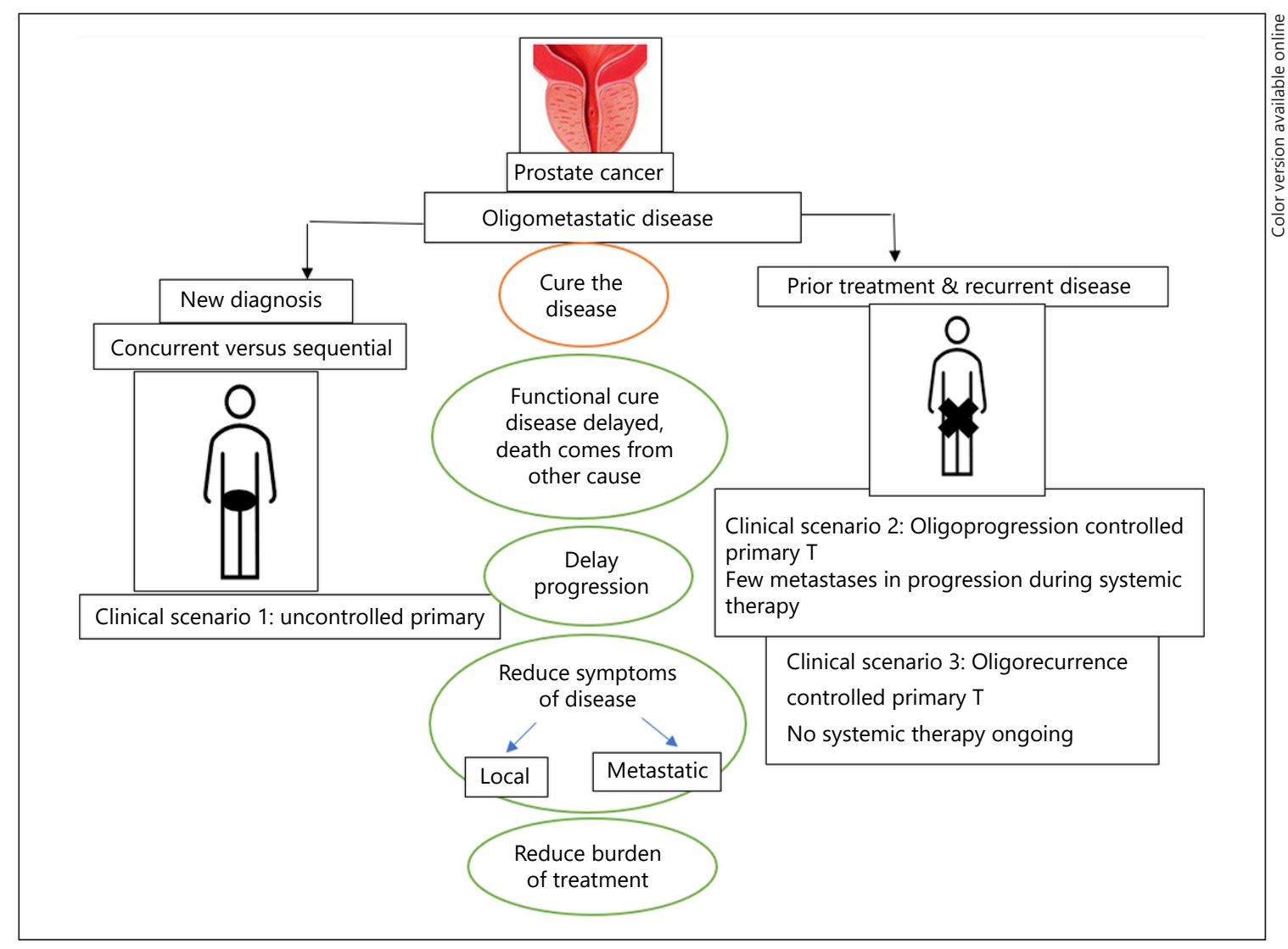

Fig. 1. Oligometastatic concept.

clude liver, lung, and brain lesions; and include lesions contained within the axial versus the appendicular skeleton $[11,12]$. In this case, the patient was diagnosed with de novo M1 PCa and met "low-volume" criteria.

\section{Metastasis-Directed Therapy: Rationale}

\section{Clinical Scenario 2}

A patient was treated with a radical prostatectomy 6 years ago and underwent salvage radiation 4 years ago. Nine years later, the patient developed a bone metastasis in a rib, which was biopsy-proven. His PSA was $8.74 \mathrm{ng} /$ $\mathrm{mL}$. He was treated with radiation therapy to the left posterior ninth rib plus 1-year of combined ADT. After stopping the hormonal therapy, the patient's PSA never recurred. He is now 59 years old with hypertension but is otherwise healthy with no other significant comorbidities.

Oligometastatic recurrence following local treatment of $\mathrm{PCa}$ is an increasingly frequent disease finding. Oligopro- gression is the most common disease state captured by most studies today. If oligoprogression is a pathway to wide-spread M1 disease, then local therapies can alter this path and may slow the disease course. Early ablation of tumors will delay adverse outcomes, including development of symptoms, especially with skeletal metastases for boneonly disease. Early intervention delays the development of castration resistance, as well as the need to start or continue systemic therapies. One example in the radiation realm is stereotactic ablative radiation, also known as stereotactic body radiation therapy (SABR/SBRT). This technique involves highly focused radiation concentrated on limited-volume tumors, with the goal of applying minimal radiation to the surrounding tissue. Four prospective trials have been published in this population. In a multicenter randomized phase II study, patients with oligometastatic PCa were randomly assigned (1:1) to either surveillance (no intervention) or to metastasis-directed surgery or SABR. The median ADT-free survival was 13 months for the surveillance group and 21 months for the metastasisdirected therapy group (MTD) (HR, 0.60, log-rank $p=$ 
0.11) [13]. The 5-year ADT-free survival was $8 \%$ for the surveillance group and $34 \%$ for the MDT group (HR = 0.57 , log-rank $p=0.06$ ). The 5 -year castration-resistant PCa-free survival was $53 \%$ for the surveillance group and $76 \%$ for the MDT group (HR 0.62; log-rank $p=0.27$ ). Prostate cancer-related mortality is low within the first 5 years of diagnosis of oligorecurrent PCa. The ORIELE study randomly assigned patients (2:1) to either observation only or to receive SABR to M1 sites outside of the prostate. Progression was observed in 19\% of patients treated with SABR versus in $61 \%$ of those in the observation arm $(p=$ 0.005) [14]. Treatment with SABR improved the median progression-free survival (PFS) (not reached vs. 5.8 months; HR, 0.30 ; $95 \% \mathrm{CI}, 0.11-0.81 ; p=0.002)$. In a prospective clinical trial, 33 patients with oligometastatic $\mathrm{PCa}$ received SABR to a total of 50 oligometastases [15]. Twenty patients had bone-only disease, 12 had node-only disease, and 1 had mixed disease. Over one-third of patients did not progress and were free from ADT at 2 years. The SABR-COMET study randomly assigned patients (1:2) to receive either palliative standard of care treatments alone (control group) or standard of care plus SABR to all M1 lesions (SABR group) [16]. The majority of these patients had 1-3 sites of disease. In a 2:1 randomization, the median OS was significantly improved for patients who underwent SABR compared to palliative approaches. The median OS was 28 months in the control group versus 41 months in the SABR group $(p=0.090)$. A nearly 2 -fold greater rate of patients were alive in the SABR arm at 5 years: $46 \%$ in the SABR arm versus $24 \%$ in the control group. Most notably, the SABR patients maintained their quality of life. When imaging identifies exclusive nodal recurrent PCa, salvage lymph node dissection can be a safe MDT option in nodal recurrence after primary treatment. However, the oncological impact of salvage lymph node dissection assessed by strong clinical endpoints remains uncertain [17]. The benefit of metastasis-directed surgery and radiotherapy (RT) to asymptomatic sites of extrapelvic disease is under active investigation and is currently somewhat unclear. In this case, the patient does meet "oligorecurrent disease," and both systemic and local treatment of all lesions is generally recommended in this scenario.

\section{Treatment of the Primary Tumor in the Setting of Oligometastatic Disease}

\section{Clinical Scenario 3}

A 55-year-old male has lower back pain. A subsequent PSA is found to be $35 \mathrm{ng} / \mathrm{mL}$. Biopsy revealed Gleason 8
PCa in 6 of 6 cores. A whole-body MRI and bone scan demonstrated a few retroperitoneal lymph nodes and 2 bone metastases in the ribs and pelvis. This patient is healthy with no significant comorbidities. What is the most appropriate next step in the care of this patient?

Cytoreductive treatment in M1 PCa refers to control of the primary tumor by radical prostatectomy, RT, or via metastasectomy at any disease site. There is also discussion surrounding the abscopal effect, a systemic antitumor immune response that reflects the regression of nonirradiated M1 lesions that are at a distance from the primary site of irradiation. It is thought that radiation induces an increased immune response via increased antigen presentation. What is unique about radiation, however, is its synergy with ADT, which increases vascularization. In the tumor microenvironment, where hypoxia is a known mechanism of radiation resistance, increased vascularization can be an incredibly powerful benefit. Androgen signaling can also decrease tumor DNA repair. DNA repair in the tumor is another mechanism of radiation resistance, so $\mathrm{ADT}$ helps the effects of radiation. A total of 8,185 M1 PCa patients were identified with the following treatment histories: no surgery or radiation therapy $(n=7,811)$, radical prostatectomy $(n=245)$, and brachytherapy $(n=129)$ [18]. Patients who had surgery and/or radiation had a lower cancer-specific mortality rate than patients who received no local therapy. The 5 -year OS and disease-specific survival were each significantly higher in patients undergoing radical prostatectomy $(67.4 \%$ and $75.8 \%$, respectively) or RT (52.6 and $61.3 \%$, respectively), than those in patients who had no surgery or radiation therapy $(22.5 \%$ and $48.7 \%$, respectively) ( $p<0.001)$. Rusthoven et al. [19] used the National Cancer Database to evaluate the OS of men with M1 PCa treated with ADT with and without prostate RT. Over 6,382 men with M1 PCa were selected, including 5,844 patients $(91.6 \%)$ receiving ADT alone and 538 (8.4\%) receiving $\mathrm{ADT}$ and RT. A total of 69 patients with M1 PCa treated with prostatectomy plus ADT were also identified in this study [19]. In this large contemporary analysis, men with M1 PCa who received prostate RT and ADT lived substantially longer than men treated with ADT alone. Radical prostatectomy was associated with a $52 \%$ decrease in the risk of PCa-specific mortality. Intensity-modulated radiation therapy was associated with a $62 \%$ decrease in the risk of prostate-specific cancer mortality.

The 2 most prominent studies that were done prospectively to analyze this issue are the HORRAD trial and the STAMPEDE trial. The HORRAD trial is a multicenter, 




Fig. 2. Advances in PET offer greater detection for PSA-recurrent disease. PET, positron-emission tomography; PSA, prostate-specific antigen.

randomized trial that recruited 432 patients with primary bone M1 PCa. Patients were randomized to either ADT with EBRT (RT group) or to ADT alone (control group) [20]. There was no maximum number of metastases as part of the exclusion criteria, and this was not an oligometastatic-only trial. This trial compared ADT to ADT with EBRT to the prostate, in patients with primary bone M1 PCa, and it did not show a significant difference in OS. The median OS was 45 months (95\% CI, 40.4-49.6) in the RT group and 43 months (95\% CI: 32.6-53.4) in the control group $(p=0.4)$. Furthermore, there were no subgroups with a statistically significant improvement, whether analyzed by Gleason score stage, age, or number of lesions. The HORRAD trial was negative, but RT was only $70 \mathrm{~Gy}$, which is considered a low dose, and only the prostate was treated not the nodes. Patients had incredibly heavy tumor burdens with PSA $>20 \mathrm{ng} / \mathrm{mL}$ and highvolume disease. The STAMPEDE RT trial randomized 2,061 men with newly diagnosed M1 PCa. Prostate RT was given in combination with hormonal therapy with or without docetaxel versus the standard of care, ADT alone [21]. Patients were divided into 2 groups. The first group was considered to have a high M1 burden and included patients with at least 1 extra-axial lesion and/or the presence of visceral metastases and at least 4 bone lesions. All other assessable patients were considered to have low M1 burden. RT improved failure-free survival but not OS. RT improved failure-free survival and the 3-year OS in the low-burden group, whereas RT had no benefit in the high-burden group. The low-burden group did have an OS benefit. The meta-analysis aimed to assess the effects of adding prostate RT to ADT in men with $\mathrm{mCSPC}$. There was no evidence that the addition of prostate RT to ADT improved survival $(\mathrm{HR}=0.92, p=0.78)$. The PFS results based on all men included in the study also did not provide clear evidence that prostate RT extended PFS (HR = $0.94, p=0.238$ ) [22]. The Advanced Prostate Cancer Consensus Conference (APCCC) guidelines of 2019 showed 
that the majority of expert panelists do consider radical local therapy to be appropriate for newly diagnosed oligometastatic PCa [23].

A total of 23 patients with M1 PCa (with 3 or fewer bone lesions) undergoing cytoreductive radical prostatectomy (CRP) were compared to 38 men with M1 PCa treated with ADT without local therapy [24]. Clinical PFS and cancer-specific survival were improved with CRP, and CRP effectively prevented complications of the lower and upper urinary tract. In a prospective series, $43 \mathrm{PCa}$ patients with low-volume bone metastases (1-3 lesions) undergoing CRP were compared to 40 patients receiving the best systemic therapy [25]. There was no significant oncological benefit found in the CRP group (overall and castrate resistance-free survival). There was, however, a significant reduction in locoregional complications for patients undergoing CRP (7\% vs. 35\%). A retrospective case series comprising 106 patients with newly diagnosed M1 PCa examined perioperative outcomes [26]. CRP for men with locally resectable, distant M1 PCa appeared safe and feasible. Complication rates related to CRP were not higher than when radical prostatectomy was performed for standard indications, and CRP avoided complications related to local progression. RT to the primary tumor is emerging as a promising treatment option in low-volume mCSPC. Surgery, however, is not yet a valid option. Continued assessment of the genomic and clinicopathological characteristics to further refine subsets will be important.

\section{Advanced Imaging Will Improve Outcomes in Patients with Low-Volume M1 Disease}

\section{Clinical Scenario 4}

A 54-year-old male has newly diagnosed high-grade PCa. His screening PSA was $15.6 \mathrm{ng} / \mathrm{mL}$, and his staging scans showed an isolated solitary bone metastasis. We recommended systemic therapy alone, thinking this patient has M1 disease. A prostate-specific membrane antigen (PSMA) positron-emission tomographic (PET) imaging was done, which showed uptake in the actual tumor, but no uptake in the bone island. This suggested that perhaps it was not true bony disease and that this patient was not oligometastatic and can potentially be cured. He underwent a radical prostatectomy and remains with an undetectable PSA.

It is important to remember that conventional imaging is used to define the oligometastatic state in retrospective studies and in most contemporary studies. The National Comprehensive Cancer Network (NCCN) defines staging guidelines for newly diagnosed PCa and suggests that individuals who present with unfavorable intermediate-risk PCa or high- or very-high-risk PCa should be staged with a conventional bone scan, CT, and MRI. More recently, the NCCN has included PET-based imaging for better resolution and more accurate diagnostic staging of the bone. However, the inclusion of PET-based imaging in the NCCN guidelines and in most clinical practice guidelines is limited to imaging for recurrences (Fig. 2) [27-32]. Thus, PET-based imaging is recommended for individuals who have had their primary $\mathrm{PCa}$ treated with surgery or radiation and subsequently developed a recurrence. With the increased sensitivity of novel imaging, the rate of diagnosis of oligometastatic disease has increased. A meta-analysis that focused on the impact of gallium-68-PSMA on the management of PCa patients with biochemical recurrence showed that it altered the management of more than half of PCa patients with a biochemical recurrence [33]. It is important to factor in this increased specificity into diagnoses and to remember that some patients who we think have metastases may not. In the clinical scenario above, the patient does meet M1 disease criteria with conventional imaging, but gallium68-PSMA does not show M1 disease. It is essential to take into consideration the clinical history of the patients to interpret the imaging results. PSMA PET will soon be a tool used in treatment decision-making for most disease stages.

\section{Considerations for Systemic Therapy in Optimal Management of Oligometastatic Disease}

\section{Clinical Scenario 5}

A 73-year-old male presented with $\mathrm{PCa}$, but there are negative findings on conventional imaging. He has Gleason $4+4$ PCa diagnosed in 6 of 12 cores on a recent biopsy. His PSA was $13.99 \mathrm{ng} / \mathrm{mL}$ prior to the biopsy. He has hypertension and is otherwise healthy and takes no other medications. He is treated with 24 months of combined ADT and 78 Gy intensity-modulated radiation therapy. At 12 months, the PSA is $0.8 \mathrm{ng} / \mathrm{mL}$; at 18 months, $2.6 \mathrm{ng} / \mathrm{mL}$; at 24 months, $3.2 \mathrm{ng} / \mathrm{mL}$; and now at 30 months, following intensity-modulated radiation therapy, the PSA is $5.4 \mathrm{ng} / \mathrm{mL}$. Restaging CT and a bone scan demonstrated M1 disease in 3 ribs, the sternum, and the scapula. The patient remains asymptomatic and otherwise in excellent health. What would the next most appropriate step be in the treatment of this patient? 
M1 PCa is a different biological entity than a primary tumor [34]. Primary PCas often harbor multiple morphologically and clonally distinct tumor foci, even though after treatment many subclones are eliminated [35-37]. The lethal clones that were present in the primary tumor will persist in the metastases. MDT may delay ADT, but outcomes are likely better with ADT as ADT in combination with radiation helps to more effectively treat the primary or M1 tumor. ADT is also likely treating the occult M1 disease that we cannot detect with current technologies. The European Organization for Research and Treatment of Cancer (EORTC) did a randomized phase 3 trial assessing the benefit of adding long-term androgen suppression with a luteinizing hormone-releasing hormone agonist to external irradiation in patients with $\mathrm{PCa}$ with high M1 risk [38]. The 10-year clinical disease-free survival was $23 \%$ in the RT alone group and $48 \%$ in the combined treatment group (HR 0.42, $p<0.0001$ ). The ten-year OS was $40 \%$ in patients receiving RT alone and $58 \%$ in those randomized to the combined treatment (HR $0.60, p=0.0004$ ). The 10 -year prostate cancer mortality was $30 \%$ and $10 \%$, respectively (HR $0.38,95 \%, p<$ 0.0001 ). This is commonly touted as a way to improve radiation therapy, but really, it is a way to attain systemic control of the disease. When ADT plus radiation is given for localized disease in the high-risk setting, the combination could help reduce the primary tumor volume and help achieve systemic control of the disease. Thus, ADT is the standard of care for men with locally advanced PCa. ADT is the standard of care in $\mathrm{M} 1 \mathrm{PCa}$, whether given as $\mathrm{ADT}$ alone or as $\mathrm{ADT}$ with abiraterone or chemotherapy. Some patients with oligometastatic PCa show rapid progression at multiple sites, some progress slowly, and others never progress. A surveillance strategy is the therapeutic recommendation in the absence of symptoms and an observed slow rise in PSA levels, whereas androgen suppression is the recommendation in the case of rapidly progressive and symptomatic disease. Furthermore, docetaxel-based chemotherapy combined with ADT has demonstrated superiority in randomized trials. However, treatment toxicity and reduction in quality of life are main issues when treating patients with oligometastatic disease. Many questions remain regarding the management of patients that present with nonsymptomatic oligometastatic PCa. Treatment for these patients would follow the paradigm that is currently most prominent in PCa oncology: integrate new therapies in earlier stages of disease rather than reserving them for the later stages [39]. With docetaxel, there was no OS benefit in low-volume patients in the CHAART-

Management of Oligometastatic Disease in Prostate Cancer
ED study. In fact, in the APCCC guidelines of 2019, a consensus panel of experts suggested that docetaxel for oligometastatic disease was not essential and should not be considered a vital component of the treatment [40]. In the LATITUDE trial involving men with high-risk, newly diagnosed $\mathrm{mCSPC}$, the rate of OS was significantly higher among those who received ADT plus abiraterone and prednisone than among those who received ADT plus placebo (median survival 53.3 vs. 36.5 months, HR 0.66, 95\% CI 0.56-0.78) [41]. In the STAMPEDE study, the addition of abiraterone to ADT did confer a significant advantage in terms of PFS and OS, even in patients with lower volume disease [42]. The STAMPEDE study demonstrated that the M1 status did confer some degree of importance in terms of the outcome in patients receiving $\mathrm{ADT}$ plus abiraterone versus $\mathrm{ADT}$ alone. This suggests that in many patients with lower volume disease, the addition of abiraterone may be beneficial. In later analyses of men with MCSPC, coadministration of abiraterone with ADT was beneficial irrespective of "risk" or "volume" [43-45]. Regulators and purchasers could consider extending the approval of abiraterone in M1 PCa to all patients. ARCHES is a multinational, doubleblind phase III trial in which 1,150 patients with mCSPC were randomized (1:1) to enzalutamide plus ADT or placebo plus ADT, stratified by disease volume [46]. Enzalutamide plus ADT significantly reduced the risk of radiographic disease progression or death by $61 \%$ compared with placebo plus ADT (HR, 0.39; 95\% CI, 0.30-0.50; $p<0.001)$. The treatment effect of enzalutamide plus ADT was independent of low-volume disease and prior docetaxel chemotherapy. The double-blind phase III TITAN clinical trial in mCSPC randomly assigned patients to ADT plus either apalutamide ( $240 \mathrm{mg}$ daily) or placebo [47]. The benefit was seen both in men with highvolume and low-volume M1 disease. An ongoing study, the Alliance Foundation trial (AFT-19), is enrolling patients with rising PSA after radical prostatectomy with no metastases on conventional imaging [48], as well as patients who have low-volume pelvic lymph node disease and patients who have PSMA PET evidence of M1 disease. In this study, a small number of patients will be treated with systemic therapy alone as there are 3 arms: 1 is ADT alone, 1 is ADT plus apalutamide, and the third is $\mathrm{ADT}$ plus apalutamide plus abiraterone. The addition of apalutamide with or without abiraterone acetate/prednisone, compared with $\mathrm{ADT}$ alone, will prolong disease suppression and potentially eradicate micrometastatic disease with a finite duration of treatment in patients with biochemically relapsed PCa. When considering sys- 


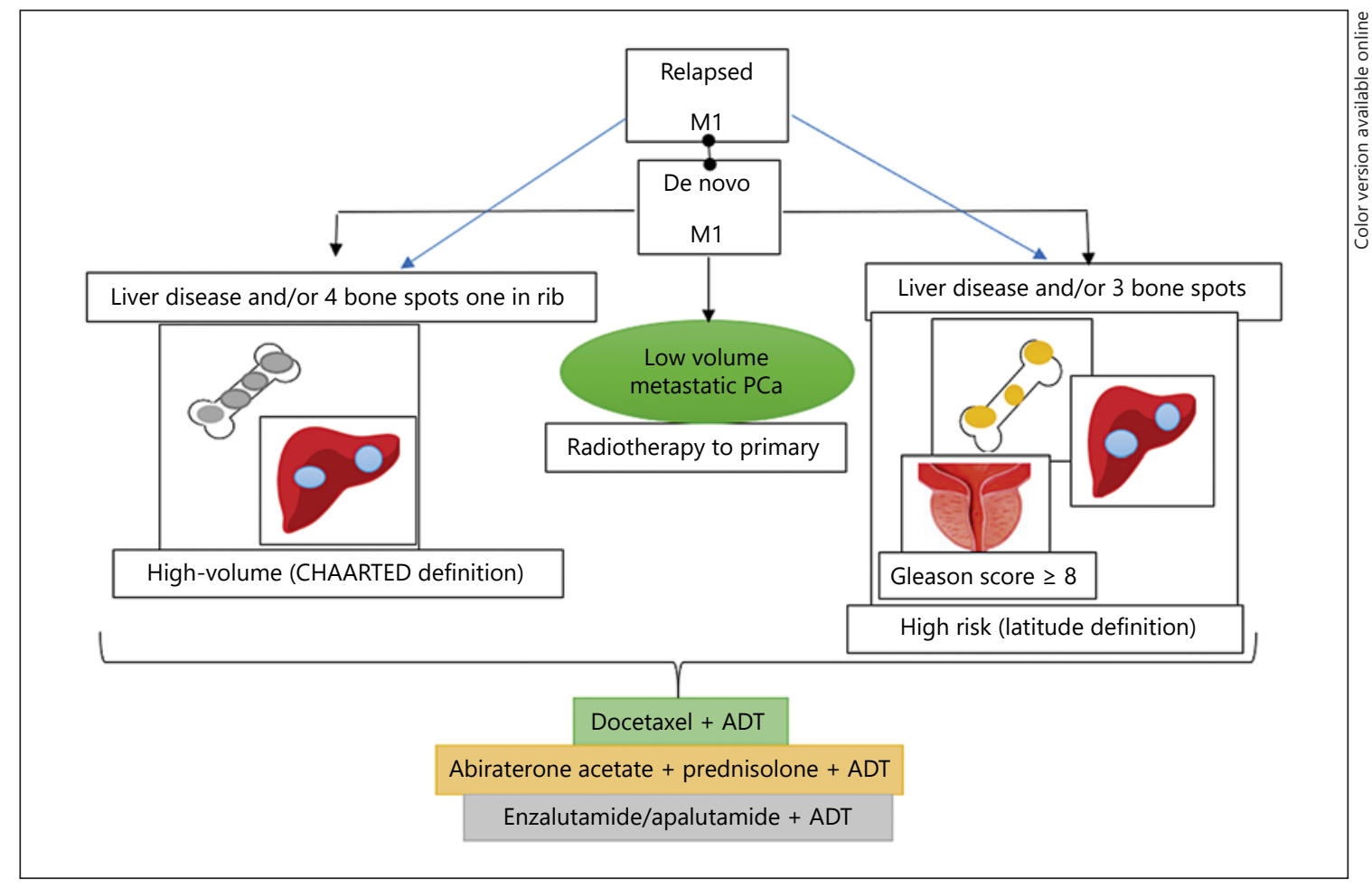

Fig. 3. Clinical drivers of decision-making. PCa, prostate cancer; M1, metastatic; ADT, androgen deprivation therapy.

temic therapies, we should consider oligometastatic disease as having a different biology than the more advanced M1 cases. We do not advocate for docetaxel in this setting as it has not been proven to be beneficial. However, as emerging studies are showing, abiraterone, enzalutamide, apalutamide, and perhaps darolutamide, as well as other drugs, may be applicable in the oligometastatic disease setting (PFS, OS, and QOL) (Fig. 3). Treatment decision-making for $\mathrm{PCa}$ is complex for both patients and physicians, so it is essential to consider the individual patient and the different disease characteristics.

\section{Conclusion}

Oligometastatic disease is not a new concept, but it is a "new" clinical state and remains relatively poorly understood. This idea elicits great interest and hope among clinicians, researchers, and patients. Finally, there is very little data to guide treatment decisions when considering oligometastatic PCa. Oligometastatic disease will be redefined as novel imaging tools continue to be adopted as it is an individual, heterogeneous entity with distinct M1 phenotypes and wide prognos- tic variability. Future studies should aim to provide clinicians with guidance on how to better tailor personalized treatment regimens.

\section{Statement of Ethics}

This study does not involve ethical issues.

\section{Conflict of Interest Statement}

All authors declare no conflict of interest.

\section{Funding Sources}

No funding received for this work.

\section{Author Contributions}

B.C. contributed to the research conception and design. B.C. and C.W. contributed to the data analysis and interpretation. C.W and O.G. drafted this paper. O.G. supervised the study. All authors approved the final version of this work. 


\section{References}

1 Siegel RL, Miller KD, Jemal A. Cancer statistics, 2018. CA Cancer J Clin. 2018 Jan;68(1): 7-30.

2 Kelly SP, Anderson WF, Rosenberg PS, Cook $\mathrm{MB}$. Past, current, and future incidence rates and burden of metastatic prostate cancer in the United States. Eur Urol Focus. 2018 Jan; 4(1):121-7.

3 Hellman S, Weichselbaum RR. Oligometastases. J Clin Oncol. 1995 Jan;13(1):8-10.

4 Weichselbaum RR, Hellman S. Oligometastases revisited. Nat Rev Clin Oncol. 2011 Jun; 8(6):378-82.

5 Soloway MS, Hardeman SW, Hickey D, Raymond J, Todd B, Soloway S, et al. Stratification of patients with metastatic prostate cancer based on extent of disease on initial bone scan. Cancer. 1988 Jan 1;61(1):195-202.

6 Ost P, Jereczek-Fossa BA, As NV, Zilli T, Muacevic A, Olivier K, et al. Progression-free survival following stereotactic body radiotherapy for oligometastatic prostate cancer treatment-naive recurrence: a multi-institutional analysis. Eur Urol. 2016 Jan;69(1):912.

7 Gandaglia G, Karakiewicz PI, Briganti A, Passoni NM, Schiffmann J, Trudeau V, et al. Impact of the site of metastases on survival in patients with metastatic prostate cancer. Eur Urol. 2015 Aug;68(2):325-34.

8 Francini E, Gray KP, Xie W, Shaw GK, Valença L, Bernard B, et al. Time of metastatic disease presentation and volume of disease are prognostic for metastatic hormone sensitive prostate cancer (mHSPC). Prostate. 2018 Sep;78(12):889-95.

9 Sridharan S, Steigler A, Spry NA, Joseph D, Lamb DS, Matthews JH, et al. Oligometastatic bone disease in prostate cancer patients treated on the TROG 03.04 RADAR trial. Radiother Oncol. 2016 Oct;121(1):98-102.

10 Tangen CM, Hussain MH, Higano CS, Eisenberger MA, Small EJ, Wilding G, et al. Improved overall survival trends of men with newly diagnosed M1 prostate cancer: a SWOG phase III trial experience (S8494, S8894 and S9346). J Urol. 2012 Oct;188(4): 1164-9.

11 Sweeney CJ, Chen YH, Carducci M, Liu G, Jarrard DF, Eisenberger M, et al. Chemohormonal therapy in metastatic hormone-sensitive prostate cancer. N Engl J Med. 2015 Aug 20;373(8):737-46.

12 Fizazi K, Tran N, Fein L, Matsubara N, Rodriguez-Antolin A, Alekseev BY, et al. Abiraterone plus prednisone in metastatic, castration-sensitive prostate cancer. $\mathrm{N}$ Engl J Med. 2017 Jul 27;377(4):352-60.

13 Ost P, Reynders D, Decaestecker K, Fonteyne V, Lumen N, De Bruycker A, et al. Surveillance or metastasis-directed therapy for oligometastatic prostate cancer recurrence: a prospective, randomized, multicenter phase II trial. J Clin Oncol. 2018 Feb 10;36(5):44653.
14 Phillips R, Shi WY, Deek M, Radwan N, Lim SJ, Antonarakis ES, et al. Outcomes of observation vs stereotactic ablative radiation for oligometastatic prostate cancer: the ORIOLE phase 2 randomized clinical trial. JAMA Oncol. 2020 May 1;6(5):650-9.

15 Siva S, Bressel M, Murphy DG, Shaw M, Chander S, Violet J, et al. Stereotactic abative body radiotherapy (SABR) for oligometastatic prostate cancer: a prospective clinical trial. Eur Urol. 2018 Oct;74(4):455-62.

16 Palma DA, Olson R, Harrow S, Gaede S, Louie AV, Haasbeek C, et al. Stereotactic ablative radiotherapy versus standard of care palliative treatment in patients with oligometastatic cancers (SABR-COMET): a randomised, phase 2, open-label trial. Lancet. 2019 May 18; 393(10185):2051-8.

17 Ploussard G, Gandaglia G, Borgmann H, de Visschere P, Heidegger I, Kretschmer A, et al. Salvage lymph node dissection for nodal recurrent prostate cancer: a systematic review. Eur Urol. 2019 Oct;76(4):493-504.

18 Culp SH, Schellhammer PF, Williams MB. Might men diagnosed with metastatic prostate cancer benefit from definitive treatment of the primary tumor? A SEER-based study. Eur Urol. 2014 Jun;65(6):1058-66.

19 Rusthoven CG, Jones BL, Flaig TW, Crawford ED, Koshy M, Sher DJ, et al. Improved survival with prostate radiation in addition to androgen deprivation therapy for men with newly diagnosed metastatic prostate cancer. J Clin Oncol. 2016 Aug 20;34(24):2835-42.

20 Boeve LMS, Hulshof M, Vis AN, Zwinderman AH, Twisk JWR, Witjes WPJ, et al. Effect on survival of androgen deprivation therapy alone compared to androgen deprivation therapy combined with concurrent radiation therapy to the prostate in patients with primary bone metastatic prostate cancer in a prospective randomised clinical trial: data from the HORRAD trial. Eur Urol. 2019 Mar 75(3):410-8.

21 Parker CC, James ND, Brawley CD, Clarke NW, Hoyle AP, Ali A, et al. Radiotherapy to the primary tumour for newly diagnosed, metastatic prostate cancer (STAMPEDE): a randomised controlled phase 3 trial. Lancet. 2018 Dec 1;392(10162):2353-66.

22 Burdett S, Boevé LM, Ingleby FC, Fisher DJ, Rydzewska LH, Vale CL, et al. Prostate radiotherapy for metastatic hormone-sensitive prostate cancer: a STOPCAP systematic review and meta-analysis. Eur Urol. 2019 Jul; 76(1):115-24

23 Gillessen S, Attard G, Beer TM, Beltran H, Bjartell A, Bossi A, et al. Management of patients with advanced prostate cancer: report of the advanced prostate cancer consensus conference 2019. Eur Urol. 2020 Apr;77(4): 508-47.

24 Heidenreich A, Pfister D, Porres D. Cytoreductive radical prostatectomy in patients with prostate cancer and low volume skeletal metastases: results of a feasibility and casecontrol study. J Urol. 2015 Mar;193(3):8328.

25 Steuber T, Berg KD, Røder MA, Brasso K, Iversen $\mathrm{P}, \mathrm{Huland} \mathrm{H}$, et al. Does cytoreductive prostatectomy really have an impact on prognosis in prostate cancer patients with low-volume bone metastasis? Results from a prospective case-control study. Eur Urol Focus. 2017 Dec;3(6):646-9.

26 Sooriakumaran P, Karnes J, Stief C, Copsey B, Montorsi F, Hammerer P, et al. A multi-institutional analysis of perioperative outcomes in 106 men who underwent radical prostatectomy for distant metastatic prostate cancer at presentation. Eur Urol. 2016 May;69(5):78894.

27 Eiber M, Maurer T, Souvatzoglou M, Beer AJ, Ruffani A, Haller B, et al. Evaluation of hybrid 68Ga-PSMA ligand PET/CT in 248 patients with biochemical recurrence after radical prostatectomy. J Nucl Med. 2015 May;56(5): 668-74.

28 Nanni C, Zanoni L, Pultrone C, Schiavina R, Brunocilla E, Lodi F, et al. (18)F-FACBC (antil-amino-3-(18)F-fluorocyclobutane-1-carboxylic acid) versus (11)C-choline PET/CT in prostate cancer relapse: results of a prospective trial. Eur J Nucl Med Mol Imaging. 2016 Aug;43(9):1601-10.

29 Schwenck J, Rempp H, Reischl G, Kruck S, Stenzl A, Nikolaou K, et al. Comparison of 68Ga-labelled PSMA-11 and 11C-choline in the detection of prostate cancer metastases by PET/CT. Eur J Nucl Med Mol Imaging. 2017 Jan;44(1):92-101.

30 Andriole GL, Kostakoglu L, Chau A, Duan F, Mahmood U, Mankoff DA, et al. The impact of positron emission tomography with $18 \mathrm{~F}$ fluciclovine on the treatment of biochemical recurrence of prostate cancer: results from the LOCATE trial. J Urol. 2019 Feb;201(2):32231.

31 Rousseau E, Wilson D, Lacroix-Poisson F, Krauze A, Chi K, Gleave M, et al. A prospective study on 18F-DCFPyL PSMA PET/CT imaging in biochemical recurrence of prostate cancer. J Nucl Med. 2019 Nov;60(11): 1587-93.

32 Eiber M, Kroenke M, Wurzer A, Ulbrich L, Jooss L, Maurer T, et al. 18F-rhPSMA-7 PET for the detection of biochemical recurrence of prostate cancer after radical prostatectomy. J Nucl Med. 2020 May;61(5):696-701.

33 Diao W, Cao Y, Su D, Jia Z. Impact of 68 Gallium prostate-specific membrane antigen tracers on the management of patients with prostate cancer who experience biochemical recurrence. BJU Int. 2021 Feb;127(2):153-63.

34 Fleischmann A, Rocha C, Schobinger S, Seiler R, Wiese B, Thalmann GN. Androgen receptors are differentially expressed in Gleason patterns of prostate cancer and down-regulated in matched lymph node metastases. Prostate. 2011 Apr;71(5):453-60. 
35 Arora R, Koch MO, Eble JN, Ulbright TM, Li L, Cheng L. Heterogeneity of Gleason grade in multifocal adenocarcinoma of the prostate. Cancer. 2004 Jun 1;100(11):2362-6.

36 Clark J, Attard G, Jhavar S, Flohr P, Reid A, De-Bono J, et al. Complex patterns of ETS gene alteration arise during cancer development in the human prostate. Oncogene. 2008 Mar 27;27(14):1993-2003.

37 Lindberg J, Klevebring D, Liu W, Neiman M, $\mathrm{Xu}$ J, Wiklund P, et al. Exome sequencing of prostate cancer supports the hypothesis of independent tumour origins. Eur Urol. 2013 Feb;63(2):347-53.

38 Bolla M, Van Tienhoven G, Warde P, Dubois JB, Mirimanoff RO, Storme G, et al. External irradiation with or without long-term androgen suppression for prostate cancer with high metastatic risk: 10-year results of an EORTC randomised study. Lancet Oncol. 2010 Nov; 11(11):1066-73.

39 Kyriakopoulos CE, Chen YH, Carducci MA, Liu G, Jarrard DF, Hahn NM, et al. Chemohormonal therapy in metastatic hormonesensitive prostate cancer: long-term survival analysis of the randomized phase III E3805 CHAARTED trial. J Clin Oncol. 2018 Apr 10; 36(11):1080-7.
40 Gillessen S, Attard G, Beer TM, Beltran H, Bjartell A, Bossi A, et al. Management of patients with advanced prostate cancer: report of the Advanced Prostate Cancer Consensus Conference 2019. Eur Urol. 2020 Apr;77(4): 508-47.

41 Fizazi K, Tran N, Fein L, Matsubara N, Rodriguez-Antolin A, Alekseev BY, et al. Abiraterone acetate plus prednisone in patients with newly diagnosed high-risk metastatic castration-sensitive prostate cancer (LATITUDE): final overall survival analysis of a randomised, double-blind, phase 3 trial. Lancet Oncol. 2019 May;20(5):686-700.

42 James ND, de Bono JS, Spears MR, Clarke NW, Mason MD, Dearnaley DP, et al. Abiraterone for prostate cancer not previously treated with hormone therapy. N Engl J Med. 2017 Jul 27;377(4):338-51.

43 Clarke NW, Ali A, Ingleby FC, Hoyle A, Amos CL, Attard G, et al. Addition of docetaxel to hormonal therapy in low- and high-burden metastatic hormone sensitive prostate cancer: long-term survival results from the STAMPEDE trial. Ann Oncol. 2019 Dec 1; 30(12):1992-2003.

44 Hoyle AP, Ali A, James ND, Cook A, Parker CC, de Bono JS, et al. Abiraterone in "high-" and "low-risk" metastatic hormone-sensitive prostate cancer. Eur Urol. 2019 Dec;76(6): 719-28.
45 James N, Rush H, Clarke N, Attard G, Cook A, Dearnaley D, et al. 6110 Abiraterone acetate plus prednisolone for hormone-naïve prostate cancer $(\mathrm{PCa})$ : long-term results from metastatic (M1) patients in the STAMPEDE randomised trial (NCT00268476). Ann Oncol. 2020;31:S509.

46 Armstrong AJ, Szmulewitz RZ, Petrylak DP Holzbeierlein J, Villers A, Azad A, et al. ARCHES: a randomized, phase III study of androgen deprivation therapy with enzalutamide or placebo in men with metastatic hormone-sensitive prostate cancer. J Clin Oncol. 2019 Nov 10;37(32):2974-86.

47 Chi KN, Agarwal N, Bjartell A, Chung BH, Pereira de Santana Gomes AJ, Given R, et al. Apalutamide for metastatic, castration-sensitive prostate cancer. N Engl J Med. 2019 Jul 4; 381(1):13-24.

48 Aggarwal RR, Eggener SE, Chen RC, Heller G, Patnaik A, Xiao $\mathrm{H}$, et al. A phase 3 study of androgen annihilation in high-risk biochemically relapsed prostate cancer: an alliance foundation trial (AFT-19). J Clin Oncol. 2018; 36. 